\title{
DNA methyltransferase $3 b$ regulates articular cartilage homeostasis by altering metabolism
}

\author{
Jie Shen, ${ }^{1}$ Cuicui Wang, ${ }^{1}$ Daofeng Li, ${ }^{2}$ Taotao Xu, ${ }^{1,3}$ Jason Myers, ${ }^{4}$ John M. Ashton, ${ }^{4,5}$ Ting Wang, ${ }^{2}$ \\ Michael J. Zuscik, ${ }^{6}$ Audrey McAlinden, ${ }^{1,7}$ and Regis J. O'Keefe ${ }^{1}$ \\ 'Department of Orthopaedic Surgery and 'Department of Genetics, Center for Cenome Sciences and Systems Biology, \\ School of Medicine, Washington University, St. Louis, Missouri, USA. ${ }^{3}$ Institute of Orthopaedics and Traumatology, the \\ First Affiliated Hospital of Zhejiang Chinese Medical University, Hangzhou, China. ${ }^{4}$ Cenomics Research Center, School \\ of Medicine and Dentistry, and ${ }^{5}$ Department of Microbiology and Immunology, School of Medicine and Dentistry, and \\ ${ }^{6}$ Department of Orthopaedics, School of Medicine and Dentistry, University of Rochester, Rochester, New York, USA. \\ 'Department of Cell Biology \& Physiology, School of Medicine, Washington University, St. Louis, Missouri, USA.
}

Osteoarthritis (OA) is the most common form of arthritis worldwide. It is a complex disease affecting the whole joint but is generally characterized by progressive degradation of articular cartilage. Recent genome-wide association screens have implicated distinct DNA methylation signatures in OA patients. We show that the de novo DNA methyltransferase (Dnmt) 3b, but not Dnmt3a, is present in healthy murine and human articular chondrocytes and its expression decreases in OA mouse models and in chondrocytes from human OA patients. Targeted deletion of $\mathrm{Dnmt} 3 \mathrm{~b}$ in murine articular chondrocytes results in an early-onset and progressive postnatal OA-like pathology. RNA-Seq and methyIC-Seq analyses of Dnmt3b loss-of-function chondrocytes show that cellular metabolic processes are affected. Specifically, TCA metabolites and mitochondrial respiration are elevated. Importantly, a chondroprotective effect was found following Dnmt3b gain of function in murine articular chondrocytes in vitro and in vivo. This study shows that Dnmt3b plays a significant role in regulating postnatal articular cartilage homeostasis. Cellular pathways regulated by Dnmt3b in chondrocytes may provide novel targets for therapeutic approaches to treat $\mathrm{OA}$.

Conflict of interest: The authors have declared that no conflict of interest exists.

Submitted: February 23, 2017

Accepted: May 10, 2017

Published: June 15, 2017

Reference information: JCI Insight. 2017;2(12):e93612. https:// doi.org/10.1172/ji.insight.93612.

\section{Introduction}

Osteoarthritis (OA) is the most common arthritic condition, clinically characterized by articular cartilage degeneration, subchondral bone sclerosis, synovitis, and osteophyte formation (1). OA risk factors include age, gender, obesity, prior joint injury, mechanics (e.g., joint malalignment), and inherent genetic alterations $(1,2)$. Currently, there are still no effective disease-modifying treatments for OA except pain relief medication and surgical replacement of damaged joints (3-5).

At the tissue level, disruption of cartilage homeostasis in OA results in enhanced catabolism and subsequent extracellular matrix (ECM) degradation. Many of the cellular and molecular changes in OA have been identified in studies utilizing murine models of OA or from analysis of human cartilage/chondrocytes from OA patients (6-8). As a result, there is the potential for development of new therapeutic approaches to target factors involved in inflammation, oxidative stress, and autophagy, to name a few (9). In addition to altered cellular and molecular pathways, a number of gene polymorphisms have been reported in human OA (e.g., GDF5 and $S M A D 3)(10,11)$ and there are now several, robustly replicated, significant OA loci that have been identified by large-scale genome wide association studies (GWAS) (12-14). However, many candidate gene studies for OA have identified false associations due to relatively small sample sizes. For example, one study carried out meta-analysis from 9 GWAS and showed that only 2 out of 199 potential candidate genes (COL11A1 and $V E G F$ ) were associated with OA in human patients (15). The OA loci discovered to date explain only a small fraction of the heritability of OA estimated by epidemiological studies. It is now becoming clear that some of the missing heritability may be explained by inheritance of epigenetic modifications of genomic DNA (16-18).

DNA methylation is a well-characterized epigenetic mechanism involving the addition of a methyl group $\left(\mathrm{CH}_{3}\right)$ from the methyl donor S-adenosyl methionine (SAM) to a cytosine within CpG sites to form 
5-methylcytosine $(5 \mathrm{mC})$. Methylation within promoters, enhancers, or gene bodies can significantly alter gene expression profiles $(19,20)$. Of the 3 catalytic DNA methyltransferase enzymes, DNMT3A and DNMT3B are known as the de novo methyltransferases and are predominantly responsible for the establishment of unique methylation patterns during development (21). DNMT1, however, is largely responsible for maintaining the DNA methylation signatures created by these de novo enzymes during cell division. Recent genome-wide methylation profiling studies have identified differentially methylated loci in the genome of human OA chondrocytes, thereby suggesting the involvement of DNMT-driven mechanisms in the development and/or progression of OA $(18,22-26)$. This study was designed to address the potential role of the de novo Dnmt3 enzymes in regulating murine articular cartilage homeostasis. We show that Dnmt3b loss of function is associated with an OA-like pathology in mice due, in part, to alterations in cellular catabolic and metabolic processes. Importantly, we also show reduced DNMT3B expression in human OA chondrocytes and that $D n m t 3 b$ gain-of-function mice are protected from developing OA. These findings may lead to the discovery of novel therapeutic targets to treat OA.

\section{Results}

Decreased expression of Dnmt3b is associated with a murine and human OA phenotype. To determine protein localization of the de novo Dnmt3 enzymes in murine articular cartilage, IHC staining was carried out on tissue sections of 3-month-old WT murine knee joints. Expression of Dnmt3a was undetectable in articular and growth plate cartilage (Figure 1A). However, Dnmt3b was abundantly expressed in chondrocytes of articular cartilage yet almost undetectable in chondrocytes of the underlying growth plate (Figure 1A). During murine cartilage development, Dnmt3b was ubiquitously expressed in proliferating chondrocytes of developing limbs at E18.5, while expression became restricted to articular chondrocytes during postnatal development (Supplemental Figure 1; supplemental material available online with this article; https://doi. org/10.1172/jci.insight.93612DS1).

We next examined the effects of aging and OA on Dnmt3b expression in cartilage. Analysis of an age range of articular cartilage tissue sections revealed a trend toward decreased Dnmt3b expression with increasing age as shown by 2 representative IHC images (at 3 and 27 months old) (Figure 1B). Surgical induction of OA in mice was carried out by meniscal ligament injury (MLI) (8) to destabilize the joint and induce articular cartilage degradation over time. Lower Dnmt3b expression levels were noted in articular chondrocytes 4 weeks following MLI compared with sham controls (Figure 1C). It has been reported that mice fed a high-fat diet (HFD) develop accelerated OA (27). Here we also show reduced expression of Dnmt3b in chondrocytes from mice fed a HFD for 4 weeks compared with control mice fed a normal diet (Figure 1D).

In human nondiseased articular cartilage tissue sections, abundant DNMT3B expression was consistently detected, whereas expression was either undetectable or restricted to articular chondrocytes of the deep zone. However, DNMT3B expression in cells from OA tissues ranged from undetectable to some positively labeled cells in the deeper zones of articular cartilage (Figure 1E). Decreased DNMT3B expression in OA cartilage was further confirmed by real-time quantitative PCR (qPCR) from human primary articular chondrocytes (PACs) (Figure 1F). Treatment of human PACs with the proinflammatory cytokine IL-1 $\beta$ resulted in decreased DNMT3B mRNA and protein expression (Figure 1G). These PACs were obtained from knee joints following total knee replacement surgery and were found to be responsive to IL-1 $\beta$ based on the expected changes in COL2A1 and MMP-13 expression (Supplemental Figure 2). Similarly, Dnmt3b expression was also decreased in murine PACs in response to IL-1 $\beta$ (Supplemental Figure 3, E and F). These data suggest that inflammatory mediators (known to increase in the context of OA) may regulate DNMT3B. In fact, we identified an NF- $\mathrm{BB}$ binding site in the promoter region of the murine Dnmt3b gene (also present in the human DNMT3B promoter) (Supplemental Figure 3A). Luciferase reporter assays showed functional utilization of the NF- $\mathrm{KB}$ binding site following IL-1 $\beta$ treatment of murine ATDC-5 cells; this affect was attenuated following mutation of the binding site (Supplemental Figure $3 \mathrm{~B}$ ). NF- $\kappa \mathrm{B}$ interaction with its binding site was also shown by ChIP assays (Supplemental Figure 3, C and D).

Given the trend toward decreased Dnmt3b expression with age and OA, the effect of Dnmt3b knockdown in murine PACs (Supplemental Figure 4, A and B) was examined. Knockdown resulted in decreased expression of the anabolic cartilage gene Col2a1 and increased expression of markers associated with catabolism or terminal hypertrophic chondrocyte differentiation (Col10a1, Runx2, and Mmp13) (Supplemental Figure 4C). Alkaline phosphatase (ALP) activity also increased in PACs following Dnmt3b knockdown, 
A
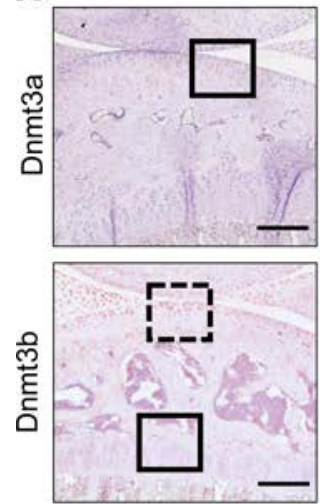

C
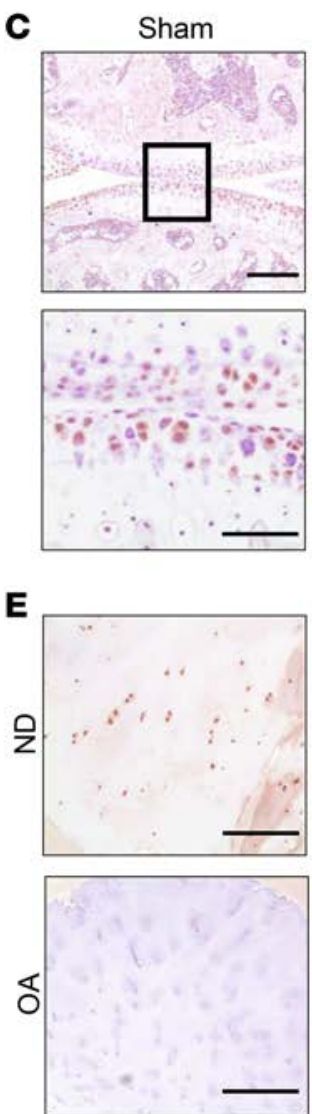
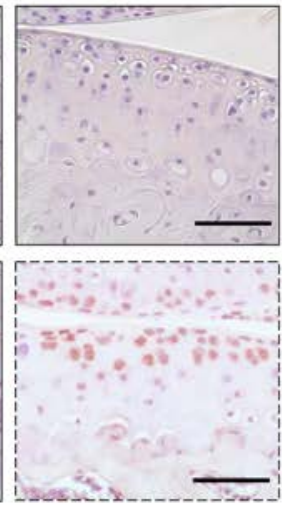

MLI
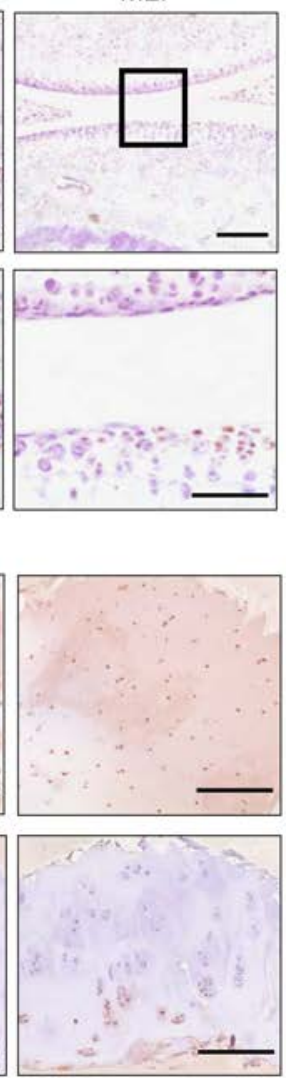

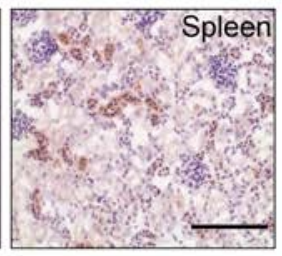

B 3-month-old
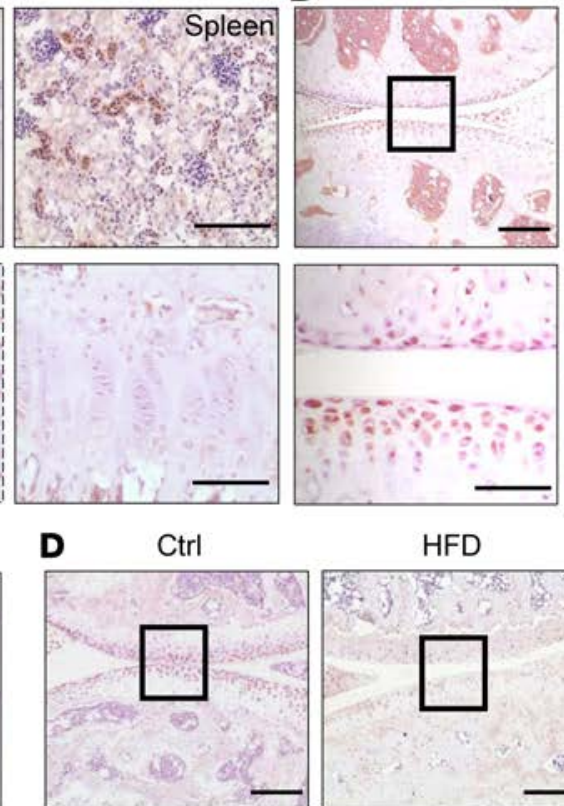

27-month-old
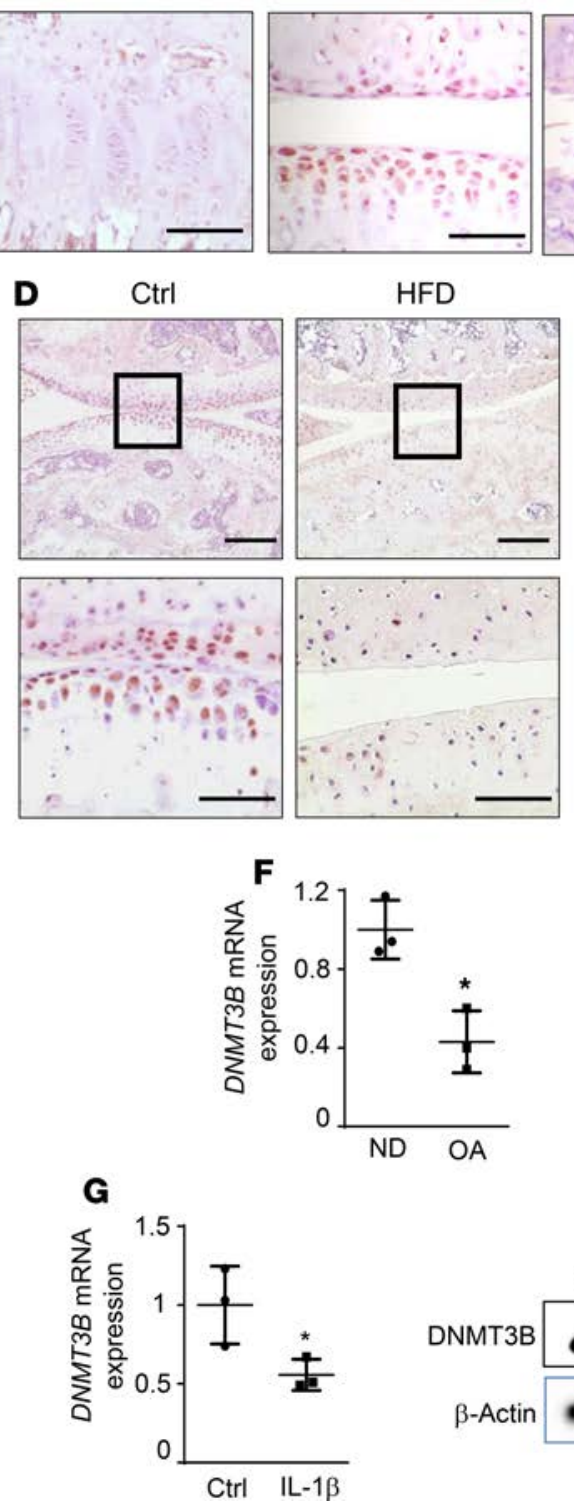
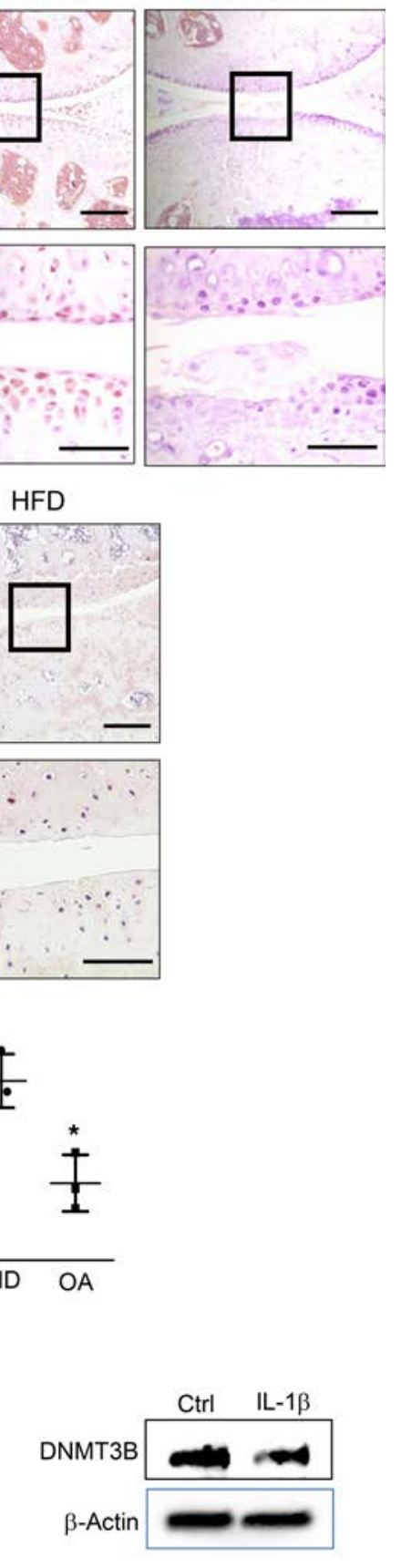

Figure 1. Dnmt3b expression in murine and human knee joint cartilage. (A) Representative images showing that Dnmt3a is not expressed in chondrocytes from 3-month-old WT articular cartilage (Dnmt3a expression in pancreas served as a positive control), while robust expression of Dnmt3b is seen in chondrocytes of 3-month-old WT articular cartilage, with lower expression in underlying growth plate cartilage $(n=3)$; the magnified images of the dashed and solid boxed areas of articular cartilage are in separate panels. (B) Representative images showing Dnmt3b expression in 3-monthold $(n=3)$ versus 27-month-old $(n=3)$ WT murine knee articular cartilage. (C) Representative images showing Dnmt3b expression in 14-week-old murine articular cartilage following meniscal ligament injury (MLI) surgery $(n=3)$ or cartilage from sham control knees $(n=3)$. (D) Representative images showing Dnmt3b expression in 14-week-old murine articular cartilage in mice fed a high-fat diet (HFD) ( $n=3)$ or controls (Ctrl) fed a normal diet $(n=3)$. (E) Two representative images showing DNMT3B expression in human healthy (ND) articular cartilage $(n=11)$ or osteoarthritic $($ OA) cartilage tissue sections $(n=71)$. (F) Reduced DNMT3B expression in human primary OA chondrocytes compared with healthy chondrocytes $(n=3)$. (G) Induction of human primary chondrocytes $(n=3)$ with IL-1 $\beta$ results in decreased expression of DNMT3B mRNA and protein levels. Scale bars: $100 \mu$ m. ${ }^{*} P<0.05$ by 2 -tailed Student's $t$ test. 
A

Ctrl

Dnmt3b LOF

Dnmt3b LOF

Dnmt3b LOF
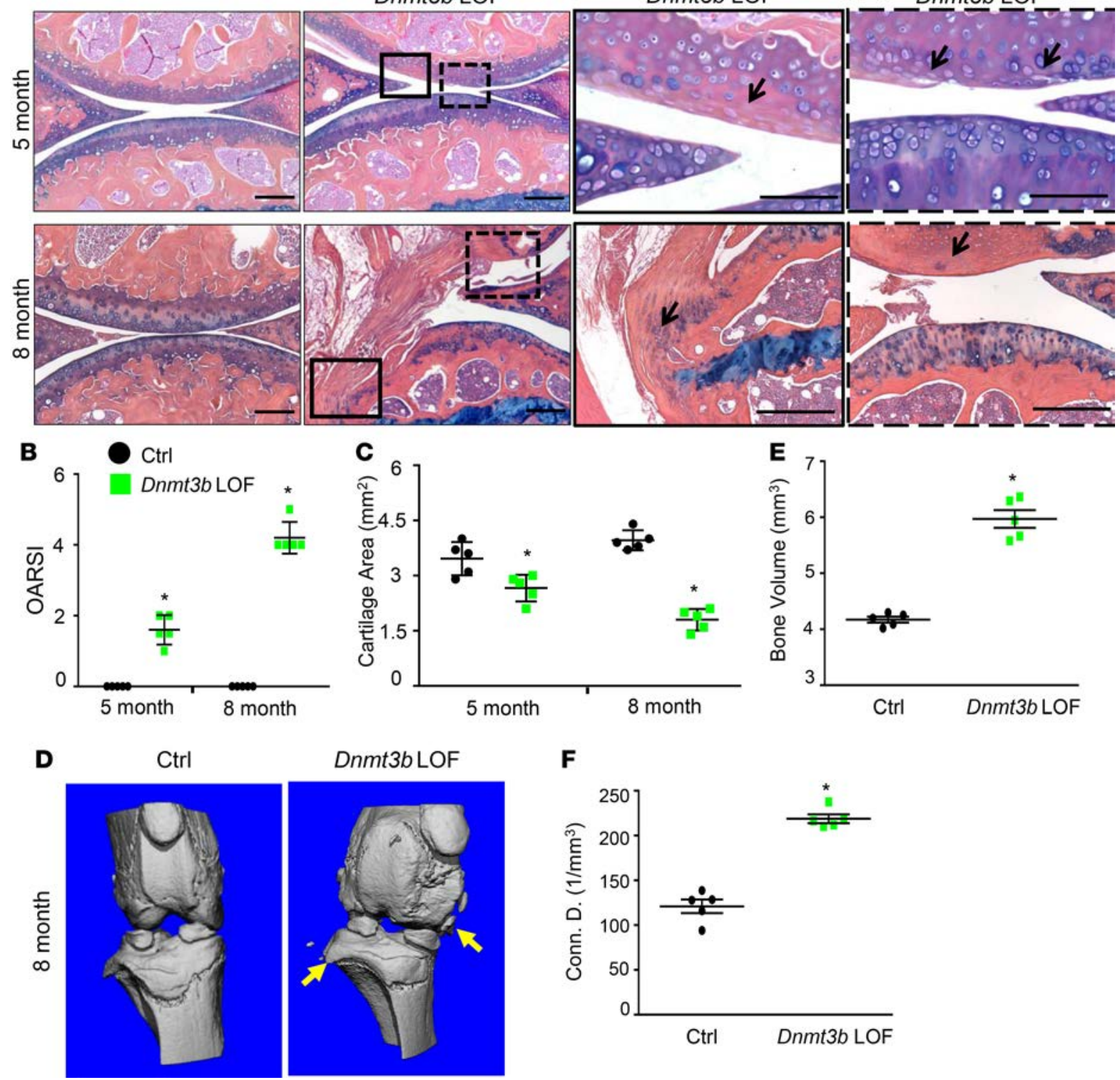

$\mathbf{F}$

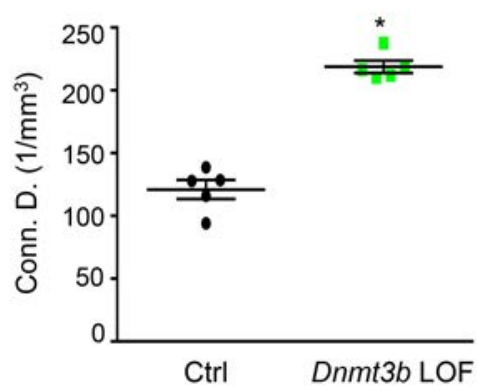

Figure 2. Dnmt3b loss-of-function mice develop accelerated osteoarthritis. (A) Representative Alcian blue/hematoxylin/orange $G$ (ABH/OC) staining of tissue sections of knee joints harvested from 5- and 8-month-old Dnmt3b loss-of-function (LOF) mice and Cre+ control (Ctrl) mice ( $n=5)$. The magnified images of the boxed regions are shown in separate panels. (B) OARSI scoring system was used to quantify the ABH/OG-stained tissue sections ( $n=5$ ). (C) Articular cartilage area was quantified by histomorphometry $(n=5)$. (D) Representative microCT images of knee joints from 8-month-old $D n m t 3 b$ LOF and Ctrl mice $(n=5)$. (E) Subchondral bone volume and $(\mathbf{F})$ subchondral bone trabecular connective density were calculated from the microCT images $(n=5)$. Arrows in 5-month-old Dnmt3b LOF mice show areas of proteoglycan loss and cartilage fibrillation. Arrows in 8-month-old Dnmt3b LOF mice show osteophyte formation and an area of proteoglycan loss in articular cartilage. Yellow arrows in microCT images denote osteophyte formation. Scale bars: $100 \mu \mathrm{m}$. ${ }^{*} P<0.05$ by ANOVA with post hoc test (B and $\mathbf{C}$ ) or 2-tailed Student's $t$ test (E and $\left.\mathbf{F}\right)$.

albeit not to the level induced by BMP-2 (Supplemental Figure 4D). Since TGF- $\beta$ and BMP signaling can have opposing effects on chondrocyte catabolism (2), Smad signaling was analyzed following Dnmt $3 b$ knockdown. Phospho-Smad2 (p-Smad2) levels decreased while p-Smad1/5 levels increased following Dnmt3b siRNA treatment (Supplemental Figure 4, E and F). This suggests that increased BMP-2 signaling may, in part, explain the increase in chondrocyte catabolism.

Dnmt 36 conditional knockdown in vivo results in a progressive OA-like pathology. To investigate the effect of Dnmt3b knockdown in vivo, transgenic mice were generated to conditionally delete Dnmt $3 b$ in cartilage using the Credriver line, $A g c C r e E R T 2$ (28). The specificity of this Cre-driver line to target chondrocytes in cartilage tissue was confirmed by crossing with Rosa-tomato $(m T / m G)$ mice (Supplemental Figure 5A). $A g c C r e^{E R T 2}$; Dnmt3 $3^{\text {h/fl}}$ mice 
were generated and injected with tamoxifen at 2 months of age to avoid the developmental defect. Tamoxifentreated $\mathrm{AgcCr} e^{E R T 2}$; Dnmt $3 b^{f / f l}$ mice will hereafter be referred to as Dnmt3b loss-of-function (LOF) mice. Supplemental Figure 5B shows an apparent reduction in Dnmt3b protein expression in articular cartilage from Dnmt3b LOF mice at 3 months of age. We then investigated if expression levels of the other Dnmt's (Dnmt3a and Dnmt1) or the recently discovered ten-eleven translocation (TET) DNA demethylation enzymes changed in Dnmt3b LOF cartilage. No significant changes in Dnmt3a, Dnmt1, Tet1, Tet2, or Tet3 expression was found (Supplemental Figure 6A). However, a small but significant decrease in overall TET activity was found (Supplemental Figure 6B). Further analysis of chondrocyte gene expression from 3-month-old Dnmt3b LOF mice revealed a significant reduction in Col2a1 expression and significant increases in Col10a1, Runx2, and Mmp13 expression (Supplemental Figure 6C).

Tissue sections from knee joints of male Dnmt $3 b$ LOF mice and male Cre ${ }^{+}$control littermates at 5 and 8 months of age were histologically assessed for signs of pathology. Compared with $\mathrm{Cre}^{+}$controls, knee joints from 5-month-old Dnmt3b LOF male mice showed features of OA including loss of proteoglycan staining in regions of articular cartilage in addition to articular cartilage tears (Figure 2A). A significant increase in the number of apoptotic cells, as determined by TUNEL staining, was found in 5-month-old Dnmt3b LOF articular cartilage (Supplemental Figure 7, A and B). In vitro analysis of Dnmt3b LOF articular chondrocytes also showed an increase in levels of reactive oxygen species (ROS) compared with control cells (Supplemental Figure 7C). At 8 months of age, histological staining revealed distorted whole-joint structure, osteophyte formation at the margin of the joint, as well as loss of proteoglycan staining (Figure 2A). Quantification of cartilage pathology using the Osteoarthritis Research Society International (OARSI) scoring system was performed by 2 blinded researchers by examining 13-16 slides per mice, and the results showed higher maximal scores for both 5- and 8-month-old Dnmt3b LOF knee joints compared with age-matched joints from $\mathrm{Cre}^{+}$ control mice (Figure 2B). Also, histomorphometric analysis showed a significant decrease in cartilage area in 5- and 8-month-old Dnmt3b LOF mice compared with age-matched $\mathrm{Cre}^{+}$controls (Figure 2C). MicroCT analysis of 8-month-old knee joints showed the presence of osteophytes (Figure 2D), and quantification of these scanned images showed an increase in subchondral bone thickness in 8-month-old Dnmt3b LOF mice compared with age-matched controls (Figure 2, E and F).

Transcriptomic and epigenomic alterations in Dnmt3b LOF chondrocytes. To investigate the mechanism by which loss of $D n m t 3 b$ in articular chondrocytes led to articular chondrocyte hypertrophy and cartilage tissue degeneration, PACs were isolated from 2-month-old $D n m t 3 b^{f / f l}$ mice and infected with either Ad5CMV-Cre (Dnmt3b LOF cells, $n=3$ ) or Ad5-CMV-GFP (control cells, $n=3$ ) for 72 hours and followed by RNA-Seq and methylC-Seq (see Methods) analyses to examine genome wide gene transcription and DNA methylation alterations in LOF chondrocytes. Loss of Dnmt3b was associated with subtle but profound DNA methylation and gene expression changes in articular chondrocytes (Figure 3 and Supplemental Figure 8). Global gene expression exhibited systematic differences between Dnmt3b LOF and control, as illustrated by their separation pattern in principle component analysis (Figure 3A) and in pairwise clustering analysis (Supplemental Figure 8A). Overall, 368 genes were found to be significantly differentially expressed (Supplemental Figure 8B); the hierarchical clustering pattern of the top 25 differentially expressed genes is shown (Figure 3B). Network analysis of the top 25 differentially expressed genes suggest they are coexpressed or colocalized (Supplemental Figure 8C). These 368 genes include members of the BMP/TGF signaling pathways (e.g., Bmp4 and Tgfbr2) as well as those involved in catabolic/hypertrophic processes (e.g., Smad2/3, Runx2, and Mmp13) (Figure 3, B and C, Supplemental Figure 8D, and supplemental differentially expressed gene list). Overall, the RNA-Seq data showing alterations in BMP/TGF signaling and catabolic/hypertrophic genes is in agreement with changes known to occur in OA as well as our own in vitro and in vivo data presented here related to Dnmt3b LOF effects in vitro and in vivo. By gene ontology enrichment analysis and ingenuity pathway analysis (IPA), functions including cell cycle process, ossification, osteoblast development, lipid metabolism, cell death, and survival comprised the top hits for these differentially expressed genes (Tables 1 and 2). These functions were highly relevant to the morphology of bone and skeleton, as well as cartilage development.

The global DNA methylation pattern followed classic bimodal distribution and did not exhibit significant differences between Dnmt3b LOF and control (Figure 3D). However, local differentially methylated regions (DMRs) were widespread. Overall 4,271 DMRs were identified, with a mean difference in methylation level of 32\% (Figure 3, E and F, and supplemental DMR list). The large majority (97\%) of these DMRs were located in introns and intergenic regions, with only $1 \%$ overlapping gene promoters 
A

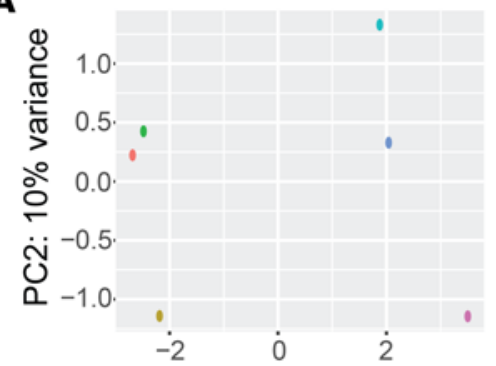

PC1: $79 \%$ variance

C

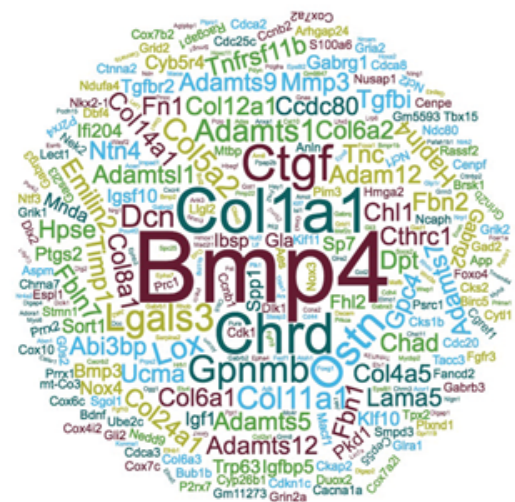

Dnmt3b LOF 1

- Dnmt3b LOF 2

- Dnmt3b LOF 3

1 Ctrl 1

- Ctrl 2

$1 \mathrm{Ctrl} 3$
B

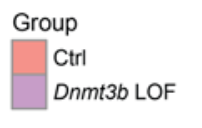

Sample

Dnmt3b LOF 1 Dnmt3b LOF 2 Dnmt3b LOF 3

Ctrl 1 Ctrl 2 Ctrl 3

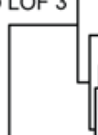

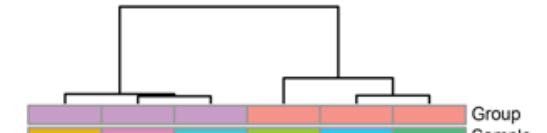

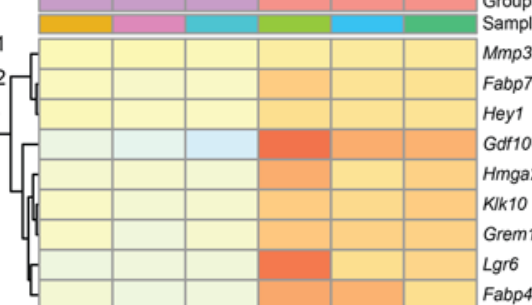

0.4

0.2

0

$-0.2$

F Differentially Expressed
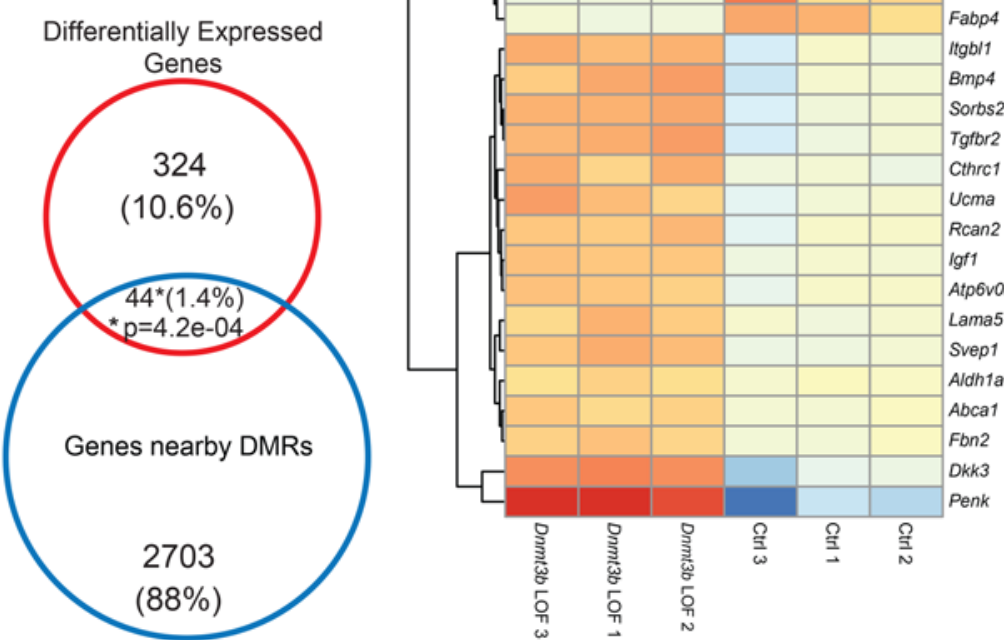

$-0.6$

D

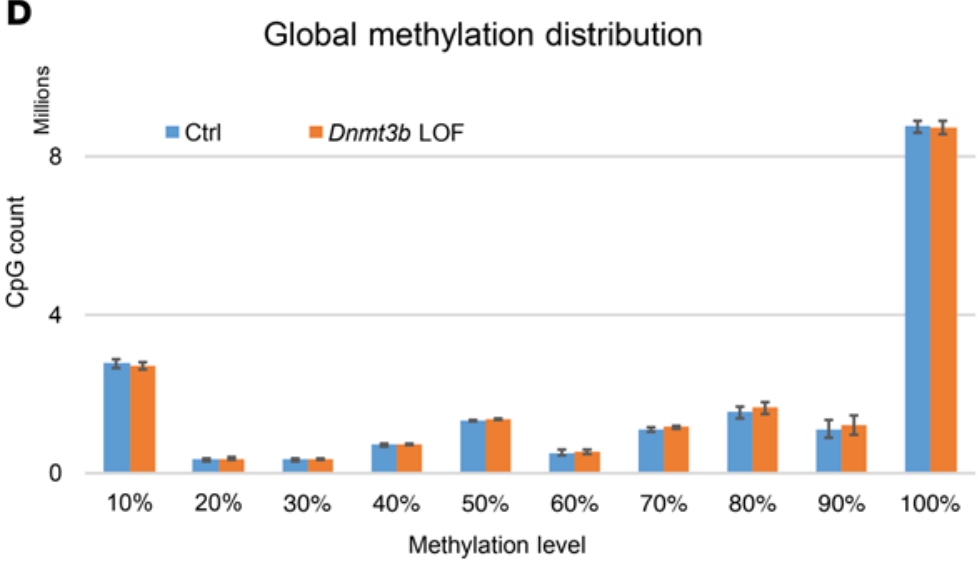

E

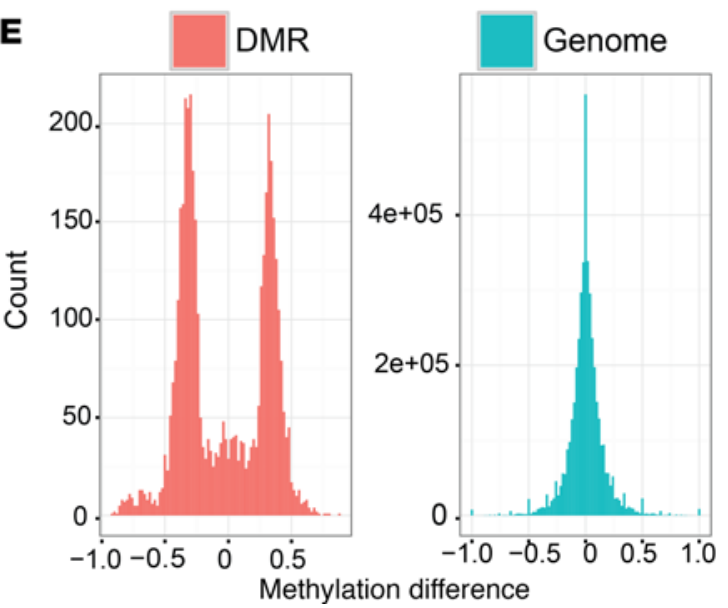

Figure 3. Altered epigenomic and transcriptomic signatures in Dnmt3b LOF chondrocytes. Cenomic DNA and RNA isolated from control and Dnmt3b loss-of-function (LOF) cells for RNA-Seq and methylC-Seq analysis $(n=3)$. (A) Principal component analysis (PCA) plot of samples based on RNA-Seq data. (B) Heatmap display of top 25 significantly differentially expressed genes between Dnmt3b LOF and control. (C) Word cloud representing gene frequency of enriched function categories from all differentially expressed genes. (D) Global methylation distribution of Dnmt3b LOF and control samples showing that there is no global difference. (E) Methylation difference of differentially methylated regions (DMRs) versus genome as background. (F) Significant overlap of genes near DMRs and differentially expressed genes. $P$ values were calculated by the hypergeometric test.

(Supplemental Figure 8E). Of all the DMRs, 44\% exhibited hypomethylation, and 56\% hypermethylation. Using GREAT (29), DMRs were associated with nearby genes; these DMR-associated genes overlapped significantly with differentially expressed genes $\left(P<6 \times 10^{-6}\right)$, and they enriched for functions related to osteoblasts and chondrocytes. One hundred and four genes exhibited changes in both expression and DNA methylation; two-thirds of these were hypomethylated, including cartilage genes that are known to be altered in OA: Ucma (30), Bmp4, and Igf1 (Figure 3, B and C). In addition, binding sites of transcription factors including Fkhr, Foxc1, Foxk1, Sox4, Sox9, and Nfatc2 were significantly 
Table 1. Function analysis from RNA-Seq

\begin{tabular}{|c|c|}
\hline Associated Network Functions & Score \\
\hline Cell Death and Survival, Cellular Assembly and Organization, Cellular Compromise & 38 \\
\hline Lipid Metabolism, Molecular Transport, Small Molecular Biochemistry & 36 \\
\hline Cell Cycle, Cellular Assembly and Organization, DNA Replication, Recombination, and Repair & 32 \\
\hline
\end{tabular}

enriched in DMRs, highlighting functional connections with pathways related to chondrogenesis (Sox4 and Sox9), inflammation (Nfatc2), and FOXO-related energy metabolism (Fkhr, Foxc1, and Foxk1) (Supplemental Figure 8F).

Increased mitochondrial metabolism in Dnmt3b LOF chondrocytes. Since mitochondria are the major organelles for lipid metabolism and energy metabolism, we investigated mitochondrial function within the context of Dnmt3b LOF. Basal and maximal mitochondrial respiration was higher in Dnmt3b LOF cells (Figure $4 \mathrm{~A})$; these findings coincided with the expected alteration in anabolic and catabolic gene expression in LOF cells (Supplemental Figure 9). Given the alterations in mitochondrial function, levels of the TCA cycle metabolites succinate and fumarate were investigated and found to be increased in Dnmt3b LOF cells compared with control cells (Figure 4B). Levels of reduced coenzyme NADH, also generated by the TCA cycle and fed into the oxidative phosphorylation pathway, were increased in Dnmt3b LOF cells (Figure 4B). Since succinate production was increased in Dnmt3b LOF cells, we then examined whether increased succinate levels coincides with increased chondrocyte catabolism/hypertrophy. Addition of succinate to WT murine PACs (Supplemental Figure 10A) resulted in increased basal and maximal respiration (Figure 4C), decreased Col2a1 expression, and an increase in the hypertrophic/catabolic chondrocyte markers Runx2 and Mmp13 (Supplemental Figure 10B). We then showed that antimycin A and rotenone (inhibitors of mitochondrial respiration, Figure 4D) could attenuate the effects of BMP-2-induced hypertrophy of WT murine PACs (Supplemental Figure 11B). These inhibitors had no effect on cell proliferation or cell death (Supplemental Figure 11A). These data strongly suggest that mitochondrial metabolism alterations coincide with chondrocyte hypertrophy and that decreased expression/function of Dnmt3b alters metabolic processes in chondrocytes undergoing hypertrophy.

Dnmt3b overexpression in vivo attenuates $O A$ progression and alters chondrocyte metabolism. Given our findings that $D n m t 3 b$ LOF in vitro and in vivo induces hypertrophic/catabolic effects in chondrocytes, we hypothesized that conditional overexpression of $D n m t 3 b$ in postnatal cartilage could have a chondroprotective effect following surgical induction of OA. Supplemental Figure 12 shows the strategy used to generate the Col2a1-Cre; Rosa-rtTA ${ }^{f /+} ;$ Dnmt3b-tg (Dnmt3b gain-of-function [GOF]) mice. In this model, addition of doxycycline (DOX) results in the expression of Dnmt3b in Col2a1-expressing cells. Supplemental Figure 13A shows the specificity of the Col2a1-Cre driver line in targeting chondrocytes in articular cartilage of Col2a1Cre; Rosa-rtTA $A^{f /+}$; H2BGFP mice, while Supplemental Figure 13B confirms Dnmt3b protein overexpression in articular chondrocytes isolated from $D n m t 3 b$ GOF mice. Therefore, this model provides both cell-specific and temporal regulation of $D n m t 3 b$ overexpression.

Following DOX administration to 10 -week-old male Dnmt3b GOF mice and $\mathrm{Cre}^{+}$control mice, MLI surgeries or sham control surgeries were carried out 24 hours later. Histological examination showed no difference in synovial joint architecture between 22-week-old Dnmt3b GOF mice and Cre ${ }^{+}$

Table 2. Enriched-pathway analysis from RNA-Seq

$\begin{array}{lc}\text { Enriched pathway } & \boldsymbol{P} \text { value } \\ \text { Hepatic Fibrosis/Hepatic Stellate Cell Activation } & 1.88 \times 10^{-9} \\ \text { Role of Osteoblasts, Osteoclasts and Chondrocytes in Rheumatoid Arthritis } & 2.87 \times 10^{-7} \\ \text { Adipogenesis Pathway } & 3.73 \times 10^{-5}\end{array}$



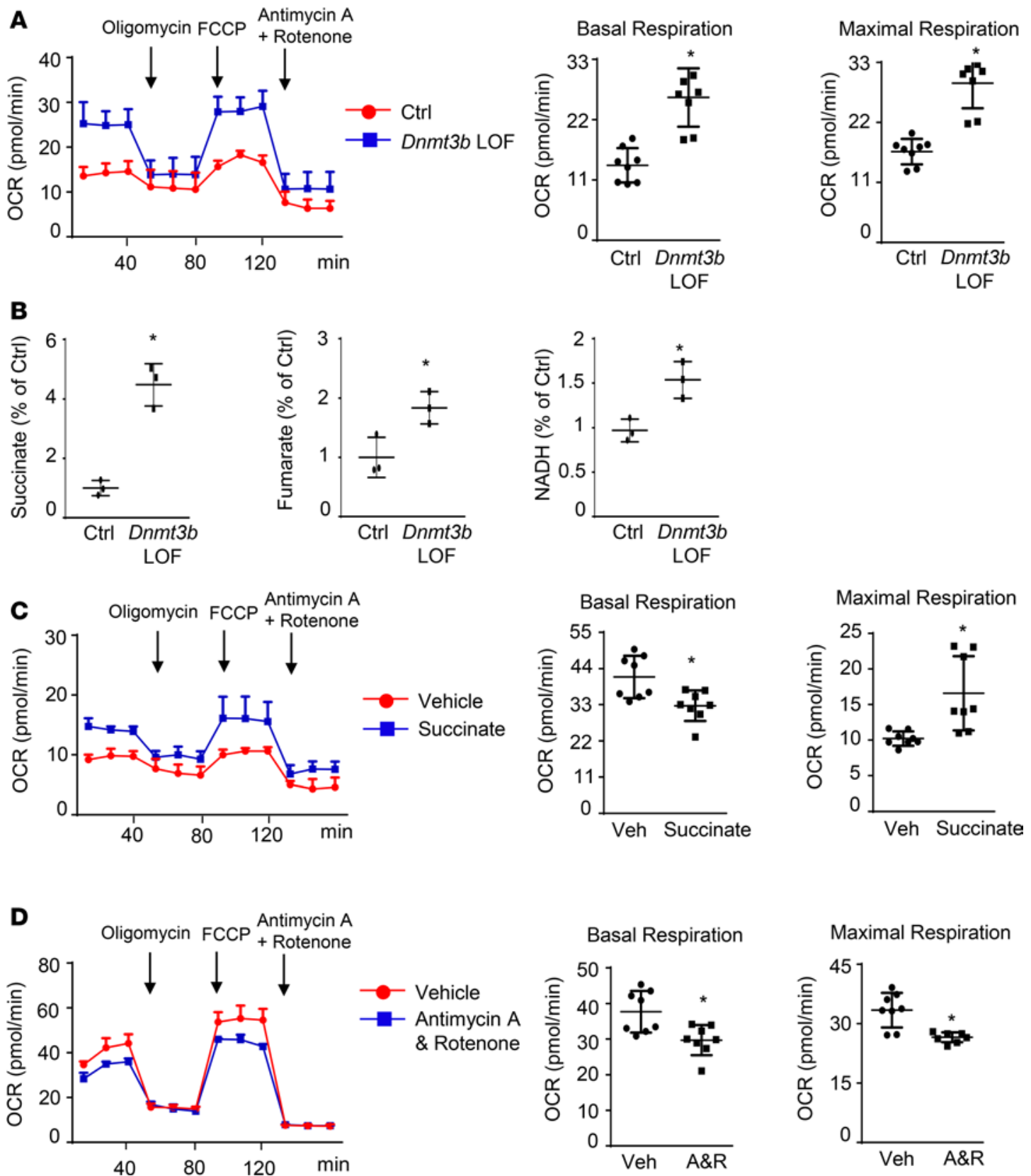

Figure 4. Mitochondrial function and cellular homeostasis in Dnmt3b LOF chondrocytes. Primary articular chondrocytes were isolated from 2-monthold Dnmt3b f/ffl mice and infected with Ad5-Cre (Dnmt3b loss-of-function [LOF]) or Ad5-GFP (Ctrl) for 48 hours. (A) Mitochondrial respiration was measured by the Seahorse XF Extracellular Flux Analyzer. Basal respiration and maximal respiration, as measured by the oxygen consumption rate (OCR) are shown $(n=8)$. (B) TCA metabolite (succinate, fumarate) and NADH analysis by HPLC-MS $(n=3)$. (C) Mitochondrial metabolism analysis was measured in 2-month-old WT cells treated with either $1 \mathrm{mM}$ diethyl succinate or vehicle (Veh) for 48 hours by the Seahorse XF Extracellular Flux Analyzer ( $n=8)$. (D) Mitochondrial respiration analysis in BMP-2-treated 2-month-old WT chondrocytes in the absence (Veh) or presence of antimycin A + rotenone (A\&R) for 48 hours $(n=8)$. ${ }^{*} P<0.05$ by 2-tailed Student's $t$ test.

control littermates in sham-operated mice (Figure 5A). However, Dnmt3b GOF mice displayed a protective effect against injury-induced OA progression at 8 and 12 weeks after MLI surgery. In general, the apparent loss of articular cartilage tissue induced by MLI was not observed in the Dnmt3b GOF mice; in fact, articular cartilage thickness was increased in the Dnmt3b GOF mice (Figure 5A). Quantification using the OARSI scoring system showed significantly lower maximal scores in the histological assessment of Dnmt3b GOF articular cartilage compared with controls at both 8 and 12 weeks (Figure 5B). Histomorphometric analyses also revealed a significant increase in articular cartilage area in Dnmt3b 
A
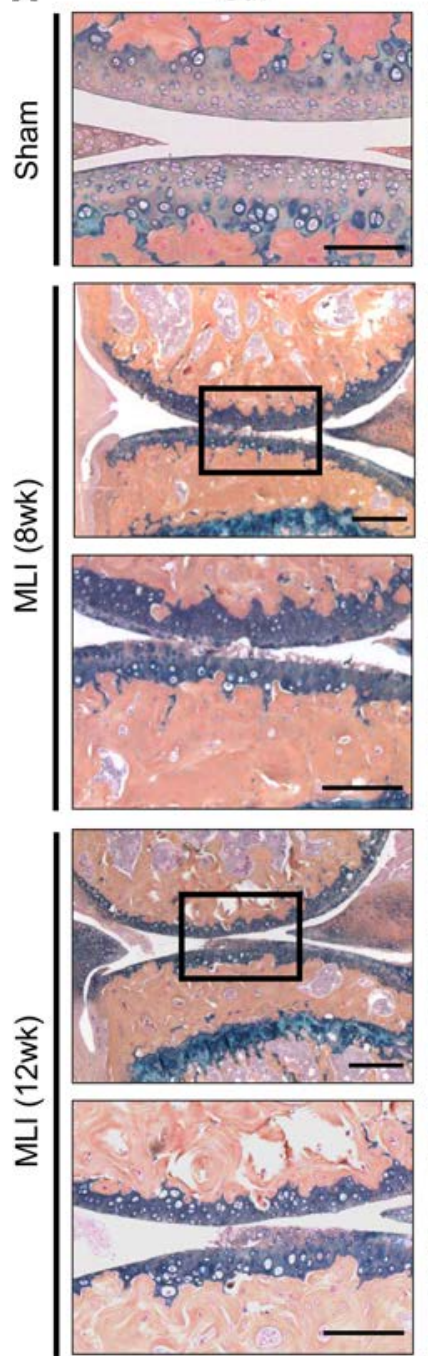
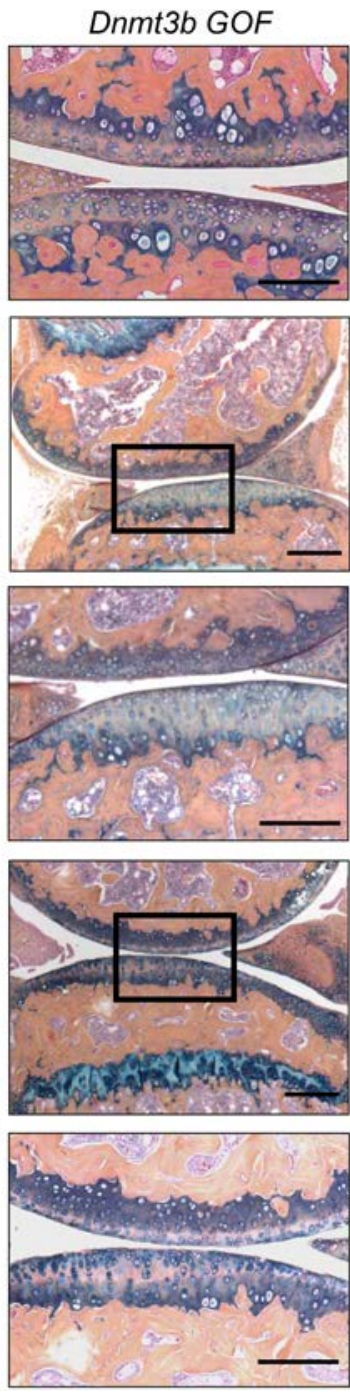
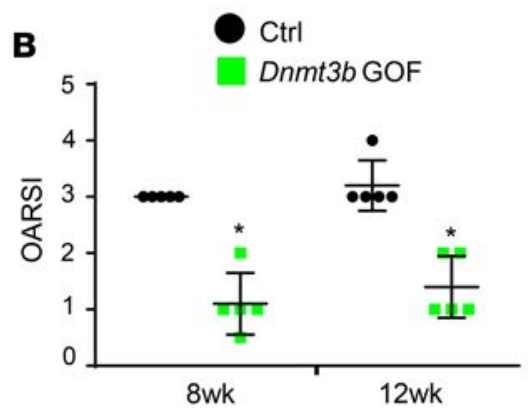

C

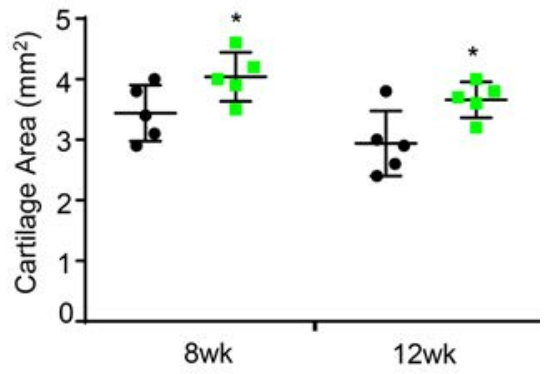

Figure 5. Dnmt3b gain-offunction mice are protected from cartilage degeneration following surgical induction of osteoarthritis. Meniscal ligament injury (MLI) or sham surgeries were performed on Dnmt3b gain-of-function (GOF) mice or $\mathrm{Cre}^{+}$control (Ctrl) mice. (A) Alcian blue/hematoxylin/ orange $G$-stained sections of Ctrl or Dnmt3b GOF knee joints 12 weeks following sham surgery. Representative images of histological sections from Ctrl or Dnmt3b GOF mice at 8 or 12 weeks following MLI surgery ( $n$ $=5$ ). The magnified images of the boxed regions are shown in separate panels. (B) Quantification of histological assessment by OARSI scoring $(n=5)$. (C) Histomorphometric analysis of Ctrl or Dnmt3b GOF cartilage $(n=5)$. Scale bars: $100 \mu \mathrm{m} .{ }^{*} P$ $<0.05$ by ANOVA followed by post hoc test.

GOF mice compared with controls (Figure 5C). Articular chondrocytes isolated from Dnmt3b GOF mice confirmed that expression of Col2a1 was significantly higher in Dnmt3b GOF cartilage, while expression of the hypertrophic markers Runx2 and Mmp 13 was reduced compared with control articular cartilage (Figure 6A).

To determine if mitochondrial metabolism was altered in Dnmt3b GOF chondrocytes, PACs from Dnmt3b GOF mice or $\mathrm{Cre}^{+}$control mice were treated with BMP-2 to induce hypertrophy. Following treatment with BMP-2, we found that the basal and maximal respiration rates of Dnmt3b GOF cells were significantly lower than in control cells (Figure 6B). These data strongly suggest that modulation of Dnmt3b levels within the context of joint injury can alter mitochondrial metabolic processes to maintain articular chondrocyte homeostasis and attenuate the progression of articular cartilage degradation.

\section{Discussion}

Recent genome-wide methylation profiling studies show that DMLs exist in DNA from OA chondrocytes compared with nondiseased control cells (18). In other studies, hypermethylation of SOX9 and COL9A1 promoters was identified in OA chondrocytes, which correlated with decreased expression of these genes $(31,32)$. Promoters of genes encoding metalloproteinases (MMP3, MMP9, MMP13, and ADAMTS4), cytokines (IL1 and IL8), sclerostin (SOST), and leptin (LEP), were shown to contain demethylated CpG sites or hypomethylation patterns, which may explain why these genes are upregulated in OA (33). From these findings, it could be hypothesized that epigenetic mechanisms involving DNMTs are responsible for disruption of chondrocyte homeostasis in OA. 
A

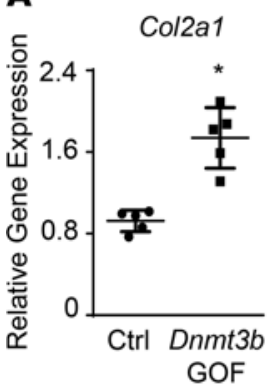

Col10a1

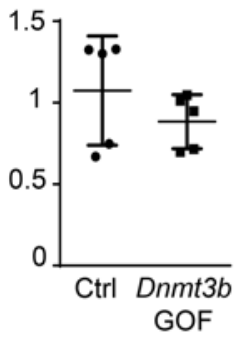

Runx2

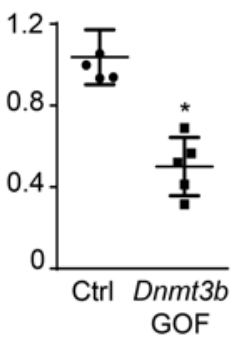

Mmp13

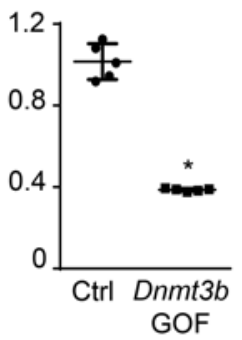

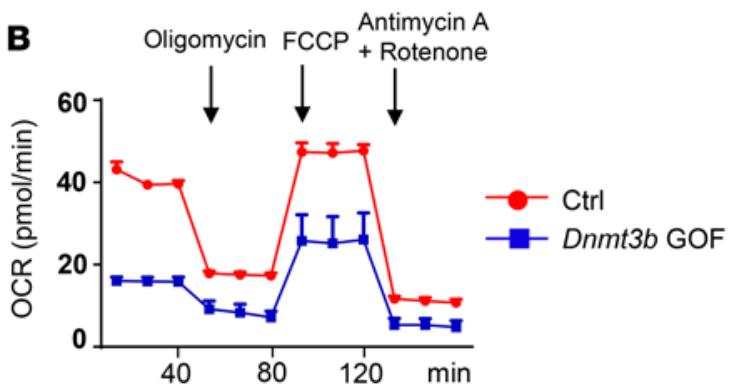
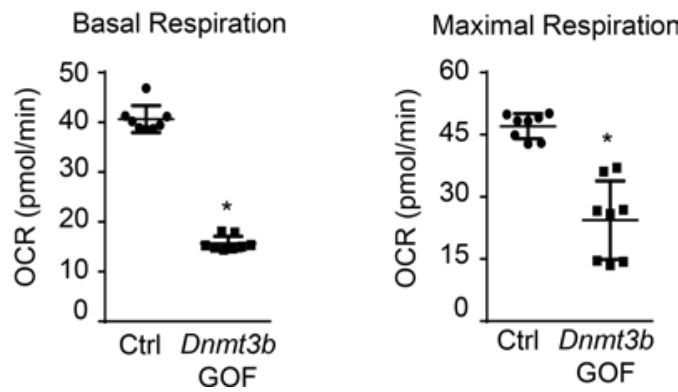

Figure 6. Mitochondrial function and cellular homeostasis in Dnmt3b GOF chondrocytes. (A) Gene expression in chondrocytes isolated from 10-weekold Ctrl or Dnmt3b gain-of-function (GOF) mice $(n=3)$. (B) Mitochondrial respiration, as measured by the oxygen consumption rate (OCR), in primary articular chondrocytes isolated from 2-month-old Col2a1Cre; Rosa-rtTA fl/+; Dnmt3b-tg mice, treated with vehicle (Ctrl) or doxycycline (Dnmt3b GOF) for 48 hours $(n=3)$. Mitochondrial respiration was measured by the Seahorse XF Extracellular Flux Analyzer. ${ }^{*} P<0.05$ by 2 -tailed Student's $t$ test.

This study focused on the de novo Dnmt's, and consistent with previous studies (34), we found that mRNA levels of Dnmt1 and Dnmt3a were 200-fold higher than that of Dnmt3b mRNA levels in murine and human articular chondrocytes. However, our studies show that Dnmt3b protein expression was much higher in cartilage tissues when compared with Dnmt3a protein. The mechanism to explain these discrepancies in mRNA and protein levels needs to be further investigated. Importantly, we showed that decreased Dnmt3b expression is associated with aging and osteoarthritic murine and human articular cartilage. To further explore the effects of decreased Dnmt3b levels at the cell and tissue level, an LOF approach was pursued in vitro and in vivo. Interestingly, Dnmt $3 b$ LOF appeared to enhance expression of markers associated with chondrocyte catabolism and terminal hypertrophic differentiation (Mmp13, Runx2, Col10a1, and $A l p)$ and alter the balance of BMP and TGF- $\beta$ signaling, favoring the BMP pathway. These cellular changes are well known to occur in chondrocytes from cartilage at various stages of OA (35). In agreement with these findings, conditional knockdown of Dnmt3b in murine cartilage in vivo was found to result in accelerated OA-like changes including cartilage fibrillation, osteophyte formation, increased ROS, and increased apoptosis. Importantly, overexpression of Dnmt3b in murine articular cartilage following surgical induction of OA appeared to be chondroprotective. Collectively, our data provide strong evidence that Dnmt3b-driven epigenetic mechanisms can regulate homeostasis of mature articular cartilage tissue.

Chronic inflammation is thought to contribute to age-related diseases such as OA $(36,37)$. Our studies show that the proinflammatory cytokine IL-1 $\beta$ downregulates Dnmt $3 b$ in murine and human chondrocytes and that these effects may be mediated via NF- $\mathrm{kB}$. Whether canonical and/or noncanonical $\mathrm{NF}-\mathrm{kB}$ signaling mechanisms play a role in regulating Dnmt3b expression has yet to be determined. In addition, IL-1 $\beta$ has also been shown to downregulate TET1 in chondrocytes $(38,39)$. TET1 belongs to the TET family of proteins that catalyze DNA demethylation, and there is growing interest in how these TET-mediated epigenetic processes control cartilage homeostasis. Recent studies have shown that TET proteins can inhibit inflammatory signals in other systems $(40,41)$. It will be interesting to elucidate if the chondroprotective effects of Dnmt $3 \mathrm{~b}$ may be due, in part, to attenuating inflammatory processes.

In addition to the expected changes in catabolic and hypertrophic gene expression due to Dnmt3b LOF, in-depth bioinformatics analyses of RNA-Seq and methylC-Seq data provided insights into other genes and pathways that may also be affected. Interestingly, analysis of differentially expressed genes in Dnmt3b LOF chondrocytes showed an enrichment in pathways involved in lipid metabolism. Lipids are important 
nutrients in chondrocyte metabolism and reports have shown a potential link between OA and disruptions in lipid metabolic processes (42-44). MethylC-Seq analysis also identified a number of transcription factor binding sites that were enriched in DMRs in Dnmt3b LOF cells. Among these were binding sites for Fhkr, Foxc1, and Foxk1, which are known to be involved in regulating a number of metabolic processes (45-47). Future studies in our laboratory are focused on identifying downstream metabolic targets of Dnmt $3 \mathrm{~b}$ and to determine potential direct links between altered metabolism and OA induction or acceleration. For the purpose of this study, we investigated if overall cellular metabolism was altered in Dnmt3b LOF cells by analysis of mitochondrial function. We found that mitochondrial respiration was increased, as were levels of TCA cycle metabolites (succinate and fumarate) and NADH (generated by the TCA cycle and fed into the oxidative phosphorylation pathway). While it is known that chondrocytes primarily utilize glycolysis for energy production, it has been shown via NMR studies that terminal hypertrophic cells rely more on oxidative phosphorylation than resting growth plate chondrocytes (48). Since we have shown that Dnmt3b LOF chondrocytes express higher levels of catabolic genes, including hypertrophic chondrocyte markers, this may be one explanation for why mitochondrial respiration appears to increase in these cells.

In conclusion, this potentially novel body of research has established that Dnmt3b-mediated mechanisms are important in maintaining postnatal cartilage homeostasis. Our findings show that inflammatory signals may play a role in decreasing Dnmt3b levels, which then leads to the disruption of a number of cellular pathways including those involved in metabolic processes. Future studies will involve deciphering what specific metabolic pathways are disrupted in a Dnmt3b LOF scenario and if direct modulation of such pathways can negatively affect chondrocyte homeostasis that may lead to an OA phenotype. Interestingly, succinate levels were found to be elevated in Dnmt3b LOF cells and recent reports have shown that succinate itself is an inflammatory signal that can induce IL-1 $\beta$ (49). It remains to be determined whether or not a vicious cycle may exist in OA chondrocytes whereby inflammation can modulate metabolic processes via downregulation of Dnmt3b, thereby leading to increased production of metabolic intermediates, such as succinate, which may then further enhance the inflammatory process. Overall, these studies will open up new avenues of research with respect to exploring alternative therapeutic strategies to treat OA. Such approaches may involve development of approaches to prevent loss of Dnmt3b activity in the OA joint or target intermediates of specific metabolic pathways that are regulated by this DNMT.

\section{Methods}

Human cartilage tissue. Two separate IRB-approved protocols were executed at the University of Rochester to collect articular cartilage from normal, OA, and injured joints. Normal cartilage was collected from amputation patients (talus or knee, $n=11$ ) and OA cartilage was harvested from total knee arthroplasty patients $(n=57)$. Injured cartilage, debrided from patients that underwent arthroscopic knee surgery to treat a recent meniscal injury (within 1 month, $n=14$ ) was collected. At harvest, tissues were immediately fixed in 10\% neutral buffered formalin and processed for embedding in paraffin. After facing the cartilage blocks on a microtome, between 1 and 4 biopsy punches were recovered from each block ( 1 mm diameter, $3 \mathrm{~mm}$ deep) and transferred to a receiver block to create a tissue microarray containing 176 biopsies. PACs were also isolated from human knee articular cartilage tissue obtained from surgeons at Washington University Department of Orthopaedic Surgery following total knee replacement surgery.

Transgenic mice. All mouse strains including Agc1Cre ${ }^{E R T 2}$ (28), Col2a1Cre (50), Dnmt3b fl/fl (51), Rosatomato $(m T / m G)(52)$, and Rosa-rtTA $A^{f l f l}$ (53) have been previously described. Dnmt3b $b^{f l f l}$ mice were generated by En Li (Novartis) and were obtained from the Mutant Mouse Regional Resource Center supported by the NIH. The $A g c 1 C r e^{E R T 2}$ mouse line was a gift from the laboratory of Benoit de Crombrugghe (MD Anderson Cancer Center). The Col2a1Cre (catalog 003554), Rosa-tomato $(\mathrm{mT} / \mathrm{m} G)$ (catalog 007576), and Rosa-rtTA $A^{f / f l}$ (catalog 006965) were purchased from the Jackson Laboratory. The efficiency of the $\mathrm{Agc1Cre} \mathrm{ERT2}^{\mathrm{E}}$ driver line in targeting chondrocytes of knee joint articular cartilage was confirmed by generation of $\mathrm{Agc1Cre} \mathrm{ERT2}^{\mathrm{E}}$; Rosa-tomato $(\mathrm{mT} / \mathrm{mG})$ mice and analysis of knee joint tissue sections by fluorescence microscopy. Agc1Cre; Dnmt3 $b^{f / f l}\left(D n m t 3 b^{A g c l E R}\right)$ mice were viable, fertile, and produced in expected Mendelian ratios. Tamoxifen ( $1 \mathrm{mg} / 10 \mathrm{~g}$ body weight/day) was delivered i.p. to all Dnmt $3 b^{A g C l E R}$ mice and $\mathrm{Cre}^{+}$littermate controls for 5 continuous days starting at 2 months of age to permanently delete the Dnmt3b gene in adult cartilage tissue. Western blot analysis of proteins extracted from articular cartilage of 3-month-old $D n m t 3 b^{A g c l E R}$ or control mice was carried out to confirm Dnmt3b knockdown in the conditional knockout mice following tamoxifen injection. Knee joints from Dnmt3b 
conditional knockout and $\mathrm{Cre}^{+}$littermate control mice were harvested at 5 and 8 months of age for histological and microCT analyses.

For production of Dnmt3b GOF mice, a construct was generated by cloning full-length Flag-tagged murine $D n m t 3 b$ cDNA into the pBI-3 vector (54). The pBI-3 vector contains an $r t$ TA promoter binding site upstream of Dnmt3b. Upon recombination, Dnmt3b is expressed with addition of Dox. Thus, the model provides both cell-specific and temporal regulation of Dnmt3b overexpression (Supplemental Figure 13). Dnmt3b-transgenic mice (Dnmt3b-tg) were subsequently generated by Cyagen Biosciences Inc. We obtained 68 Dnmt3b-tg mice, 7 of which were founder mice. Dnmt3b-tg mice were crossed with Col2a1Cre; Rosa-rtTA $A^{f /+}$ mice to generate $D n m t 3 b$ GOF mice. These mice were viable, fertile, and produced in expected Mendelian ratios. The efficiency of the Col2a1-Cre driver line in targeting chondrocytes of articular cartilage was confirmed by analysis of knee joint tissue sections of Col2a1Cre; Rosa-rtTA ${ }^{f /+}$; H2BGFP mice by fluorescence microscopy. DOX (2.5 $\mathrm{mg} / \mathrm{kg}$ body weight) was administrated i.p. to 10 -week-old male Dnmt3b GOF mice and littermate controls 1 day prior to the MLI. DOX injections continued twice per week following MLI surgery for 8 or 12 weeks, at which point mice were sacrificed and knee joint specimens harvested for downstream histological analyses.

Murine knee joint analysis. Murine hind limbs were harvested, followed by removal of skin and soft tissues before fixation in 10\% neutral-buffered formalin for 3 days and decalcification in 14\% EDTA for 7 days. Limbs were then processed, embedded in paraffin, and sectioned $(5 \mu \mathrm{m})$. Alcian blue hematoxylin/orange $\mathrm{G}$ (ABH/OG) staining was performed to analyze knee joint tissue architecture and proteoglycan localization. Quantitative histomorphometry was performed on ABH/OG-stained sections (3 slides per mice) using the Osteomeasure Analysis System (Osteometrics). The tide mark was used to differentiate between unmineralized and mineralized articular cartilages. Joint pathology was also quantified using the OARSI scoring system (55). Briefly, sagittal sections obtained every $80 \mu \mathrm{m}$ across the medial femorotibial joint were used to determine the maximal scores. For each group, 5 mice were analyzed, and 13-16 slides were examined by 2 blinded scorers. IHC was performed on sections using traditional antigen retrieval and colorimetric development methodologies. Briefly, paraffin slides were baked at $60^{\circ} \mathrm{C}$ overnight and after re-equilibration to room temperature, slides were deparaffinized through 3 changes in xylene, 100\% ethanol, 95\% ethanol, 1 change in $70 \%$ ethanol, and final rehydration in distilled water. Heat-induced antigen retrieval was performed with citric acid buffer ( $\mathrm{pH}$ 6.0). Rabbit anti-mouse antibodies against COL2A1 (Thermo Fisher Scientific, catalog MS235-P, 1:1,000), DNMT3A (LSBio, catalog LS-B7240, 1:1,000) and DNMT3B (LSBio, catalog LS-C111224, 1:1,000) were used. Impact NovaRed (Vector Laboratories, catalog SK-4805) was used for peroxidase substrate reaction. Counterstaining was performed using hematoxylin.

MicroCT analyses were performed on 2-, 4-, 6-, and 8-month-old mouse knee joints prior to decalcification using a VivaCT 40 scanner. Briefly, the tibia was scanned from the knee to the connection at the fibula using a protocol consisting of high-resolution $(10.5 \mu \mathrm{m}) \mathrm{x}$-ray energy settings of $55 \mathrm{kVp}$ and $145 \mu \mathrm{A}$ and $300 \mathrm{~ms}$ integration time. Parameters of subchondral bone volume, bone mineral density, and bone connective density were measured as previously described using the Scanco analysis software (56).

$P A C$ isolation and culture. Articular cartilage was isolated from the knee joints of 3-month-old $D n m t 3 b^{f / f l}$ mutant mice as previously described, with modifications (57). Briefly, articular cartilage was excised from femoral condyles and tibial plateaus with a scalpel and placed in $1 \times$ PBS. Cartilage fragments were digested using $3 \mathrm{mg} / \mathrm{ml}$ collagenase D in $10 \mathrm{ml}$ high glucose DMEM (Invitrogen, catalog 11965-092) for 12 hours at $37^{\circ} \mathrm{C}$. Murine articular chondrocyte cell suspensions were filtered through $0.4-\mu \mathrm{m}$ filters and seeded at a density of 500,000 cells/well in 6-well tissue culture plates in high-glucose DMEM supplemented with 10\% FBS (Atlanta Biologicals, catalog EF-0050) and 1\% penicillin/streptomycin (Life Technologies, catalog 15140-122).

Full-thickness sections of human articular cartilage were removed from the femoral condyles and tibial plateaus of specimens obtained following total knee replacement surgery, diced into 2-mm cubes, and digested in sterile spinner flasks for 90 minutes at $37^{\circ} \mathrm{C}$ in pronase digestion buffer (DMEM/F12 [Thermo Fisher Scientific, catalog 11320]; 5\% FBS [Atlanta Biologicals, catalog EF-0050], 2\% penicillin/streptomycin [Thermo Fisher Scientific, catalog 15140122], and 0.4\% pronase [Roche, catalog 11459643001]). Following washes in HBSS (Thermo Fisher Scientific, catalog 14025092), tissue was further digested in collagenase digestion buffer at $37^{\circ} \mathrm{C}$ (DMEM/F12; $5 \% \mathrm{FBS} ; 2 \%$ penicillin/streptomycin; $0.035 \%$ collagenase $\mathrm{P}$ [Roche, catalog 11213865001]) for 14 to 16 hours. Cells were collected, filtered through $70-\mu \mathrm{m}$ cell strainers (VWR, catalog 10199-656), and plated at a density of 100,000 cells/ $\mathrm{cm}^{2}$. After 24 hours, chondrocytes were treated with IL-1 $(10 \mathrm{ng} / \mathrm{ml}$; R\&D Systems, catalog 201-LB) for 48 hours. 
Next-generation RNA-Seq and data analysis. PACs were isolated from Dnmt $3 b^{f / f l}$ mice and treated with either Adeno-Cre or Adeno-GFP (MOI = 100) for 72 hours. Total RNA was collected using the AllPrep DNA/RNA Mini Kit (Qiagen, catalog 80204). Total RNA concentration was determined with the NanoDrop 1000 spectrophotometer and RNA quality was assessed with the Agilent Bioanalyzer. The TruSeq RNA Sample Preparation Kit V2 (Illumina) was used for next-generation sequencing library construction (as per the manufacturer's protocol) at the Genomics Research Center (GRC) at the University of Rochester Medical Center (URMC). Briefly, mRNA was purified from 100 ng total RNA with oligo-dT magnetic beads and fragmented. First-strand cDNA synthesis was performed with random hexamer priming followed by second-strand cDNA synthesis. End repair and 3' adenylation was then performed on the double-stranded cDNA. Illumina adaptors were ligated to both ends of the cDNA, purified by gel electrophoresis, and amplified with PCR primers specific for the adaptor sequences to generate amplicons of approximately $200-500$ bp in size. The amplified libraries were hybridized to the Illumina single-end flow cell and amplified using the cBot (Illumina) at a concentration of 8 pM per lane. Single-end reads of 100 nt were generated for each sample and aligned to the organism-specific reference genome. Sequence reads were submitted to NCBI Gene Expression Omnibus (GEO GSE85148). Raw reads generated from the Illumina HiSeq2500 sequencer were demultiplexed using configurebcl2fastq.pl version 1.8.4. Low-complexity reads and vector contamination were removed using sequence cleaner ("seqclean") and the NCBI univec database, respectively. The FASTX toolkit (fastq_quality_trimmer) was applied to remove bases with quality scores below $\mathrm{Q}=13$ from the end of each read. Processed reads were then mapped to the UCSC Mus musculus reference genome (mm10) with SHRiMP version 2.2.3 and differential expression analysis was performed using Cufflinks version 2.0.2: specifically, cuffdiff2 and usage of the general transfer format (GTF) annotation file for the given reference genome. Heatmaps of sample-to-sample distances using the rlog-transformed values, MA plots of expression changes, and heatmaps of significantly expressed genes were analyzed by DEseq2 package using R/Bioconductor platform (58). Network analysis of differentially expressed genes was generated by the GeneMania tool (http://genemania.org/). Significantly differentially expressed genes were further analyzed for statistically enriched pathways using IPA (Ingenuity Systems, http://www.ingenuity.com) and categorized for biological function using DAVID (Functional Annotation Bioinformatics, http://david.abcc.ncifcrf.gov/).

Whole-genome bisulfite sequencing and data analysis. Following treatment of murine articular chondrocytes (isolated from Dnmt3b $b^{f / f l}$ mice), with either Adeno-Cre or Adeno-GFP (MOI = 100) for 72 hours, genomic DNA was isolated using the AllPrep DNA/RNA Mini Kit. Genomic DNA was quantified using the Qubit fluorometer (Life Technologies) and DNA integrity was determined using the TapeStation with genomics DNA tape reagents (Agilent). Methylation libraries were generated with Epicentre's EpiGnome MethylSeq Kit in collaboration with the GRC at the URMC. Briefly, bisulfite conversion was carried out on 100 ng of genomic DNA using Zymo EZ DNA Methylation Gold as per the manufacturer's recommendations (Zymo Research) and conversion efficiency was determined with the Bioanalyzer 2100 (Agilent). Single-stranded cDNA was generated and tagged from bisulfite-converted DNA using random hexamers containing a $5^{\prime}$ tag followed by terminal tagging to mark the $3^{\prime}$ end of the DNA. Illumina-specific adaptors were added during PCR amplification. DNA libraries were purified with AmpureXP beads and quantified with the Bioanalyzer 2100 and Qubit fluorometer. The amplified libraries were hybridized to the Illumina paired-end flow cell and amplified using the cBot at a concentration of $8 \mathrm{pM}$ per lane. Pair end reads of $100 \mathrm{nt}$ were generated for each sample and aligned to the organism-specific reference genome. Sequences reads were also submitted to the NCBI Gene Expression Omnibus (GEO GSE85148). Raw reads generated from the Illumina HiSeq2500 sequencer were demultiplexed using configurebc12fastq.pl version 1.8.4. Pre-processing of the data was be performed using Trimmomatic-0.32 for stringent adapter removal and quality filtering. Alignment of the processed read data to the NCBI build 37.2 of the Mus musculus genome was then performed using Bismark version 0.9.0, which made use of bowtie version 2.1 .0 and samtools version 0.1.19. Methylation extraction was also performed using Bismark version 0.9.0 with the following parameters: "--cutoff 5 --ignore 3 --ignore_r2 3". A Python program was written to combine the methylation information in the form of an average percentage methylation for each locus that was called by Bismark for all 3 replicates within a group and to convert to the format that DSS package (59) required for differential methylation analysis. The DMRs were chosen using cutoffs with delta methylation level as 0.1 , $P$ value as 0.01 , and minimum length as $200 \mathrm{bp}$ (the average size of DMRs was $560 \mathrm{bp}$, data not shown). A customized Python script was used to assign methylation values to the 500-bp windows among genome, 
Table 3. Primer sequences for real-time qPCR

\begin{tabular}{|c|c|}
\hline Genes & Sequences \\
\hline Col2a1 & $\begin{array}{l}\text { 5'-GCAGAGATGGACAACCTGGTA-3' } \\
\text { 5'-AGCCTTCTCGTCATACCCT-3' }\end{array}$ \\
\hline Col10a1 & $\begin{array}{l}\text { 5'-ATGCCTTGTTCTCCTCTTACTG-3' } \\
\text { 5'-TGCTCAACGGTACCAAACG-3' }\end{array}$ \\
\hline Runx2 & $\begin{array}{l}\text { 5'-CGTCCACTCTCACTT TAATACCTC-3' } \\
\text { 5'-GTAGCCACGTTCAACGATCTG-3' }\end{array}$ \\
\hline Mmp13 & $\begin{array}{l}\text { 5'-AGACTGGTAATGCCATCAAGG-3' } \\
\text { 5'-GCCATTTCATCCTTCCTCATG-3' }\end{array}$ \\
\hline Dnmt1 & $\begin{array}{l}\text { 5'-CCTGCCAGGGCTTCAGTGGC-3' } \\
\text { 5'-CAGGCAGCGCAGTGTGAGCT-3' }\end{array}$ \\
\hline Dnmt3a & $\begin{array}{l}\text { 5'-GCCGAATTGTGTCTTGGTGGATGACA-3' } \\
\text { 5'-CCTCGTGGAATGCACTGCAGAAGGA-3' }\end{array}$ \\
\hline Dnmt3b & $\begin{array}{l}\text { 5'-AATACCCAACTCCTTCACСAC-3' } \\
\text { 5'-TCTTCACTACTCATCCTCACCT-3' }\end{array}$ \\
\hline Tet1 & $\begin{array}{l}\text { 5'-GACCCTCTTCCTCGATCTCG-3' } \\
\text { 5'-CAAACCCACCTGAGGCTGTT-3' }\end{array}$ \\
\hline Tet2 & $\begin{array}{l}\text { 5'-TGTTGTTCTCAGGGTGAGAATC-3' } \\
\text { 5'-TCTTGCTTCTGGCAAACTTACA-3' }\end{array}$ \\
\hline Tet3 & $\begin{array}{l}\text { 5'-CCGGATTGAGAAGGTCATCTAC-3' } \\
\text { 5'-AAGATAACAATCACGGCGTTCT-3' }\end{array}$ \\
\hline$\beta$-actin & $\begin{array}{l}\text { 5'-AGATGTGGATCAGCAAGCAG-3' } \\
\text { 5'-GCGCAAGTTAGGTTTTGTCA-3' }\end{array}$ \\
\hline DNMT3B & $\begin{array}{c}\text { 5'-TTGATATTCCCCTCGTCCTTC-3' } \\
\text { 5'-CGAGTCCTCTCATTCTTTCATG-3' }\end{array}$ \\
\hline COL2A1 & $\begin{array}{l}\text { 5'-GGCAATAGCAGGTTCACCTACA-3' } \\
\text { 5'-CGATAACAGTCTTCCCCCACTT-3' }\end{array}$ \\
\hline MMP13 & $\begin{array}{c}\text { 5'-CTTGACCACTCCAAGGACCC-3' } \\
\text { 5'-CCTCGACCATAGAGAGACTCGA-3' }\end{array}$ \\
\hline GAPDH & $\begin{array}{l}\text { 5'-TGTAGTTGAGGTCAATCAAGGG-3' } \\
\text { 5'-ACATCGCTCAGACACCATG-3' }\end{array}$ \\
\hline
\end{tabular}

and methylation differences between $D n m t 3 b$ LOF and control group at genome background or DMRs were plotted using ggplot2 package (http://ggplot2.org/). DMRs were further annotated to genomic regions based on refGene annotation from UCSC Genome Browser (http://genome.ucsc.edu/) using customized scripts. Motif search within DMRs was done using Homer software (60) with default parameters.

Metabolite analysis and mitochondria stress test. Proteins were isolated from PACs and 10- $\mu 1$ samples were injected into an LC-20 AD HPLC system (Shimadzu), which was coupled to a triple-quadrupole mass spectrometer (Thermo Fisher Scientific). Metabolites were analyzed by LC-MS/MS as previously described (61). Briefly, articular chondrocytes were plated in XF96 plates at 50,000 cells per well with XF Assay Medium Modified DMEM (Seahorse, catalog 101022-100). Oligomycin and FCCP (Seahorse Stress Kit) were prepared in XF assay medium at a final concentration of 5 $\mu \mathrm{M}$ and $1 \mu \mathrm{M}$, respectively, and were injected during the measurements. At the end of the assays, protein concentrations were measured for normalization.

Dnmt3b promoter luciferase assay. ATDC-5 cells were purchased from SigmaAldrich (catalog 99072806) and were transfected with a pGL4 luciferase plasmid containing cDNA encoding $1 \mathrm{~kb}$ of the murine Dnmt3b promoter using X-tremeGENE transfection reagents (Roche, catalog 06366236001). A Dnmt3b promoter construct containing mutations in the predicted NF- $\mathrm{kB}$ binding site was also transfected into an additional set of ATDC-5 cells. All transfected cells were then treated with IL-1 $\beta$ $(1 \mathrm{ng} / \mathrm{ml}$ ) for 24 hours before performing the luciferase assay.

ChIP assay. ATDC-5 cells $\left(1 \times 10^{7}\right)$ were harvested for ChIP assays following the manufacturer's protocol (Cell Signaling Technology, catalog 9003). Briefly, cells were fixed with formaldehyde and chromatin was fragmented by micrococcal nuclease to 200 to $900 \mathrm{bp}$. ChIPs were performed using an anti-NF-кB1 antibody (Cell Signaling Technology, catalog 13586, 1:50) while histone H3 and IgG antibodies were used as positive and negative controls, respectively. After reversal of protein-DNA crosslinking, the enrichment of the Dnmt3b DNA fragment during immunoprecipitation was analyzed by standard PCR and real-time qPCR. Dnmt3b primer sequences used for PCR were: Forward, 5'-CTGGTCATCTAGGAGGGTGA-3'; and Reverse, 5'-CGGGCGAGGGAGATTTG-3'.

TET activity assay. Nuclear extracts were prepared from the Dnmt3b LOF chondrocytes or WT control chondrocytes using a cell nuclear extraction preparation kit (Active Motif, catalog 40010). These nuclear extracts (containing $5 \mu \mathrm{g}$ protein) were then used to assess total TET activity according to the manufacturer's instructions (Abcam).

Real-time qPCR and Western blot. RNA was isolated from ATDC-5 chondrocytes or PACs using an RNeasy Mini kit (Qiagen, catalog 74134). cDNA synthesis and real-time RT-PCR was performed according to the manufacturers' instructions. Primer sequences for Col2a1, Runx2, Mmp13, Col10a1, Dnmt1, Dnmt3a, Dnmt3b, Tet1, Tet2, Tet3, and $\beta$-actin are shown in Table 3. Western blot analyses were conducted on the protein of lysates from either ATDC-5 cells or primary (murine or human) articular chondrocytes. The following primary antibodies were used: p-Smad1/5 (Cell Signaling Technology, catalog 9516, 1:500), p-Smad3 (Abcam, catalog ab52903, 1:1,000), Smad1/5 (Cell Signaling Technology, catalog 6944, 1:1,000), Smad3 (Abcam, catalog ab40854, 1:1,000), DNMT3A (LSBio, catalog LS-B7240, 1:1,000), DNMT3B (LSBio, catalog LS-C111224, 1:1,000) and $\beta$-actin (Sigma-Aldrich, catalog 2228, 1:2,000).

Cell proliferation and cell death detection. BMP-2-treated PACs isolated from 2-month-old WT (C57BL/6) mice were treated with vehicle or rotenone and antimycin A for 48 hours. Cell proliferation and cell death were detected by a Roche Proliferation ELISA Kit (Roche, catalog 11647229001) and Cell Death Detection ELISA Kit (Roche, catalog 11774425001).

Succinate concentration assay. PACs isolated from 2-month-old WT mice were treated with vehicle or diethylsuccinate (1 mM, Sigma-Aldrich, catalog 112402) for 48 hours. Intracellular succinate concentration was measured using the succinate colorimetric assay kit following the manufacturer's instructions (SigmaAldrich, catalog MAK184-1KT).

Statistics. Data (in vitro experiments and histomorphometry) are presented as mean \pm SD. The comparisons for OARSI scores, bone mass, and microarchitecture among different groups were performed using 
multifactorial ANOVA. When ANOVA testing indicated overall significance of main effects and without interaction between them, the difference between individual time points and sites was assessed by post hoc tests. The level of significance was set at $P$ less than 0.05 . Statistical tests for RNA-Seq and methylC-Seq data analysis were performed using the R environment (http://www.r-project.org/).

Study approval. Human cartilage tissue used for IHC analysis was harvested following the IRB-approved protocols of University of Rochester (IRB ID RSRB00042321 and ID RSRB00014518). Human knee articular chondrocyte isolation was approved by the Washington University Human Research Protection Office (IRB ID 201104119). Patients provided written informed consent prior to their participation in the study. All studies involving mice were approved by the University of Rochester Committee on Animal Resources.

\section{Author contributions}

JS conducted experiments, acquired data, analyzed data, and wrote the manuscript. CW, DL, TX, JM, and JMA conducted experiments, acquired data, and analyzed data. MJZ, TW, and AM assisted in experimental design, analyzed data, and edited the manuscript. RJO designed research studies, analyzed data, and wrote and edited the manuscript.

\section{Acknowledgments}

This work is supported by the NIH R01 grant AR069605 to RJO and AM. We would like to acknowledge the technical expertise and assistance of Sarah Mack, Kathy Maltby, and Michael Thullen within the Histology, Biochemistry, and Molecular Imaging Core in the Center for Musculoskeletal Research at the URMC.

Address correspondence to: Regis J. O'Keefe, Department of Orthopaedic Surgery, Washington University in St. Louis, 660 South Euclid, CB 8233, St Louis, Missouri 63110, USA. Phone: 314.747.8414; Email: okeefer@wudosis.wustl.edu.

1. Felson DT. Clinical practice. Osteoarthritis of the knee. NEngl J Med. 2006;354(8):841-848.

2. Wang M, Shen J, Jin H, Im HJ, Sandy J, Chen D. Recent progress in understanding molecular mechanisms of cartilage degeneration during osteoarthritis. Ann N Y Acad Sci. 2011;1240:61-69.

3. Bijlsma JW, Berenbaum F, Lafeber FP. Osteoarthritis: an update with relevance for clinical practice. Lancet. 2011;377(9783):2115-2126.

4. Anderson DD, et al. Post-traumatic osteoarthritis: improved understanding and opportunities for early intervention. J Orthop Res. 2011;29(6):802-809.

5. van den Berg WB. Osteoarthritis year 2010 in review: pathomechanisms. Osteoarthr Cartil. 2011;19(4):338-341.

6. Glasson SS, Blanchet TJ, Morris EA. The surgical destabilization of the medial meniscus (DMM) model of osteoarthritis in the 129/SvEv mouse. Osteoarthr Cartil. 2007;15(9):1061-1069.

7. Kamekura S, et al. Osteoarthritis development in novel experimental mouse models induced by knee joint instability. Osteoarthr Cartil. 2005;13(7):632-641.

8. Goldring MB, Berenbaum F. Emerging targets in osteoarthritis therapy. Curr Opin Pharmacol. 2015;22:51-63.

9. Valdes AM, et al. Genetic variation in the SMAD3 gene is associated with hip and knee osteoarthritis. Arthritis Rheum 2010;62(8):2347-2352.

10. Valdes AM, et al. The GDF5 rs143383 polymorphism is associated with osteoarthritis of the knee with genome-wide statistical significance. Ann Rheum Dis. 2011;70 (5):873-875.

11. Panoutsopoulou K, Zeggini E. Advances in osteoarthritis genetics. J Med Genet. 2013;50(11):715-724.

12. Reynard LN, Loughlin J. Insights from human genetic studies into the pathways involved in osteoarthritis. Nat Rev Rheumatol. 2013;9(10):573-583.

13. Evangelou E, et al. A meta-analysis of genome-wide association studies identifies novel variants associated with osteoarthritis of the hip. Ann Rheum Dis. 2014;73(12):2130-2136.

14. Rodriguez-Fontenla C, et al. Assessment of osteoarthritis candidate genes in a meta-analysis of nine genome-wide association studies. Arthritis Rheumatol. 2014;66(4):940-949.

15. Goldring MB, Marcu KB. Epigenomic and microRNA-mediated regulation in cartilage development, homeostasis, and osteoarthritis. Trends Mol Med. 2012;18(2):109-118.

16. Barter MJ, Bui C, Young DA. Epigenetic mechanisms in cartilage and osteoarthritis: DNA methylation, histone modifications and microRNAs. Osteoarthr Cartil. 2012;20(5):339-349.

17. Loughlin J, Reynard LN. Osteoarthritis: Epigenetics of articular cartilage in knee and hip OA. Nat Rev Rheumatol. 2015;11(1):6-7.

18. Maunakea AK, et al. Conserved role of intragenic DNA methylation in regulating alternative promoters. Nature. 2010;466(7303):253-257.

19. Gu J, et al. Mapping of variable DNA methylation across multiple cell types defines a dynamic regulatory landscape of the human genome. G3 (Bethesda). 2016;6(4):973-986.

20. Smith ZD, Meissner A. DNA methylation: roles in mammalian development. Nat Rev Genet. 2013;14(3):204-220.

21. Fernández-Tajes J, et al. Genome-wide DNA methylation analysis of articular chondrocytes reveals a cluster of osteoarthritic 
patients. Ann Rheum Dis. 2014;73(4):668-677.

22. Rushton MD, et al. Characterization of the cartilage DNA methylome in knee and hip osteoarthritis. Arthritis Rheumatol. 2014;66(9):2450-2460

23. Jeffries MA, et al. Genome-wide DNA methylation study identifies significant epigenomic changes in osteoarthritic cartilage. Arthritis Rheumatol. 2014;66(10):2804-2815.

24. Rushton MD, Young DA, Loughlin J, Reynard LN. Differential DNA methylation and expression of inflammatory and zinc transporter genes defines subgroups of osteoarthritic hip patients. Ann Rheum Dis. 2015;74(9):1778-1782.

25. Rushton MD, et al. Methylation quantitative trait locus analysis of osteoarthritis links epigenetics with genetic risk. Hum Mol Genet. 2015;24(25):7432-7444.

26. Mooney RA, Sampson ER, Lerea J, Rosier RN, Zuscik MJ. High-fat diet accelerates progression of osteoarthritis after meniscal/ligamentous injury. Arthritis Res Ther. 2011;13(6):R198.

27. Wang M, Shen J, Jin H, Im HJ, Sandy J, Chen D. Recent progress in understanding molecular mechanisms of cartilage degeneration during osteoarthritis. Ann N Y Acad Sci. 2011;1240:61-69.

28. Henry SP, Jang CW, Deng JM, Zhang Z, Behringer RR, de Crombrugghe B. Generation of aggrecan-CreERT2 knockin mice for inducible Cre activity in adult cartilage. Genesis. 2009;47(12):805-814.

29. McLean CY, et al. GREAT improves functional interpretation of cis-regulatory regions. Nat Biotechnol. 2010;28(5):495-501.

30. Lorenzo P, Bayliss MT, Heinegård D. Altered patterns and synthesis of extracellular matrix macromolecules in early osteoarthritis. Matrix Biol. 2004;23(6):381-391.

31. Kim KI, Park YS, Im GI. Changes in the epigenetic status of the SOX-9 promoter in human osteoarthritic cartilage. $J$ Bone Miner Res. 2013;28(5):1050-1060.

32. Imagawa K, et al. Association of reduced type IX collagen gene expression in human osteoarthritic chondrocytes with epigenetic silencing by DNA hypermethylation. Arthritis Rheumatol. 2014;66(11):3040-3051.

33. Barter MJ, Young DA. Epigenetic mechanisms and non-coding RNAs in osteoarthritis. Curr Rheumatol Rep. 2013;15(9):353.

34. Sesselmann S, Söder S, Voigt R, Haag J, Grogan SP, Aigner T. DNA methylation is not responsible for p21WAF1/CIP1 downregulation in osteoarthritic chondrocytes. Osteoarthr Cartil. 2009;17(4):507-512.

35. Blaney Davidson EN, et al. Increase in ALK1/ALK5 ratio as a cause for elevated MMP-13 expression in osteoarthritis in humans and mice. J Immunol. 2009;182(12):7937-7945.

36. Greene MA, Loeser RF. Aging-related inflammation in osteoarthritis. Osteoarthr Cartil. 2015;23(11):1966-1971.

37. Wang X, Hunter D, Xu J, Ding C. Metabolic triggered inflammation in osteoarthritis. Osteoarthr Cartil. 2015;23(1):22-30.

38. Taylor SE, Smeriglio P, Dhulipala L, Rath M, Bhutani N. A global increase in 5-hydroxymethylcytosine levels marks osteoarthritic chondrocytes. Arthritis Rheumatol. 2014;66(1):90-100.

39. Haseeb A, Makki MS, Haqqi TM. Modulation of ten-eleven translocation 1 (TET1), isocitrate dehydrogenase (IDH) expression, $\alpha$-ketoglutarate $(\alpha-\mathrm{KG})$, and DNA hydroxymethylation levels by interleukin-1 $\beta$ in primary human chondrocytes. $J$ Biol Chem. 2014;289(10):6877-6885.

40. Neves-Costa A, Moita LF. TET1 is a negative transcriptional regulator of IL-1 $\beta$ in the THP-1 cell line. Mol Immunol. 2013;54(3-4):264-270.

41. Zhang Q, et al. Tet2 is required to resolve inflammation by recruiting Hdac2 to specifically repress IL-6. Nature. 2015;525(7569):389-393.

42. Villalvilla A, Gómez R, Largo R, Herrero-Beaumont G. Lipid transport and metabolism in healthy and osteoarthritic cartilage. Int J Mol Sci. 2013;14(10):20793-20808.

43. Tsolis KC, et al. Comparative proteomic analysis of hypertrophic chondrocytes in osteoarthritis. Clin Proteomics. 2015;12(1):12.

44. Thijssen E, van Caam A, van der Kraan PM. Obesity and osteoarthritis, more than just wear and tear: pivotal roles for inflamed adipose tissue and dyslipidaemia in obesity-induced osteoarthritis. Rheumatology (Oxford). 2015;54(4):588-600.

45. Gross DN, van den Heuvel AP, Birnbaum MJ. The role of FoxO in the regulation of metabolism. Oncogene. 2008;27(16):2320-2336

46. Eijkelenboom A, Burgering BM. FOXOs: signalling integrators for homeostasis maintenance. Nat Rev Mol Cell Biol. 2013;14(2):83-97.

47. Kousteni S. FoxO1, the transcriptional chief of staff of energy metabolism. Bone. 2012;50(2):437-443.

48. Pollesello P, de Bernard B, Grandolfo M, Paoletti S, Vittur F, Kvam BJ. Energy state of chondrocytes assessed by ${ }^{31} \mathrm{P}-\mathrm{NMR}$ studies of preosseous cartilage. Biochem Biophys Res Commun. 1991;180(1):216-222.

49. Tannahill GM, et al. Succinate is an inflammatory signal that induces IL-1 $\beta$ through HIF-1 $\alpha$. Nature. 2013;496(7444):238-242.

50. Ovchinnikov DA, Deng JM, Ogunrinu G, Behringer RR. Col2a1-directed expression of Cre recombinase in differentiating chondrocytes in transgenic mice. Genesis. 2000;26(2):145-146.

51. Dodge JE, et al. Inactivation of Dnmt3b in mouse embryonic fibroblasts results in DNA hypomethylation, chromosomal instability, and spontaneous immortalization. J Biol Chem. 2005;280(18):17986-17991.

52. Muzumdar MD, Tasic B, Miyamichi K, Li L, Luo L. A global double-fluorescent Cre reporter mouse. Genesis. 2007;45(9):593-605

53. Belteki G, et al. Conditional and inducible transgene expression in mice through the combinatorial use of Cre-mediated recombination and tetracycline induction. Nucleic Acids Res. 2005;33(5):e51.

54. Baron U, Freundlieb S, Gossen M, Bujard H. Co-regulation of two gene activities by tetracycline via a bidirectional promoter. Nucleic Acids Res. 1995;23(17):3605-3606.

55. Glasson SS, Chambers MG, Van Den Berg WB, Little CB. The OARSI histopathology initiative - recommendations for histological assessments of osteoarthritis in the mouse. Osteoarthr Cartil. 2010;18 Suppl 3:S17-S23.

56. Sampson ER, et al. Establishment of an index with increased sensitivity for assessing murine arthritis. J Orthop Res. 2011;29(8):1145-1151.

57. Gosset M, Berenbaum F, Thirion S, Jacques C. Primary culture and phenotyping of murine chondrocytes. Nat Protoc. 2008;3(8):1253-1260.

58. Love MI, Huber W, Anders S. Moderated estimation of fold change and dispersion for RNA-seq data with DESeq2. Genome Biol. 2014;15(12):550.

59. Park Y, Wu H. Differential methylation analysis for BS-seq data under general experimental design. Bioinformatics. 
2016;32(10):1446-1453.

60. Heinz S, et al. Simple combinations of lineage-determining transcription factors prime cis-regulatory elements required for macrophage and B cell identities. Mol Cell. 2010;38(4):576-589.

61. Munger J, et al. Systems-level metabolic flux profiling identifies fatty acid synthesis as a target for antiviral therapy. Nat Biotech nol. 2008;26(10):1179-1186. 